\title{
Alter
}

Revue de phénoménologie

$21 \mid 2013$

La Vie

\section{L'enfant, l'halluciné, le primitif : figures de la raison altérée chez le premier Merleau-Ponty}

\section{Christopher Lapierre}

\section{(2) OpenEdition}

\section{Journals}

Édition électronique

URL : http://journals.openedition.org/alter/913

DOI : $10.4000 /$ alter.913

ISSN : 2558-7927

Éditeur :

Association ALTER, Archives Husserl (CNRS-UMR 8547)

\section{Édition imprimée}

Date de publication : 1 novembre 2013

Pagination : 315-335

ISBN : 978-2-95-223749-9

ISSN : $1249-8947$

\section{Référence électronique}

Christopher Lapierre, «L'enfant, l'halluciné, le primitif : figures de la raison altérée chez le premier Merleau-Ponty », Alter [En ligne], 21 | 2013, mis en ligne le 01 juin 2019, consulté le 06 juillet 2019. URL : http://journals.openedition.org/alter/913 ; DOI : 10.4000/alter.913 


\section{L'ENFANT, L'HALLUCINÉ, LE PRIMITIF : FIGURES DE LA RAISON ALTÉRÉE CHEZ LE PREMIER MERLEAU-PONTY}

Christopher Lapierre

\section{Introduction :}

L'œuvre de Merleau-Ponty tend à montrer que l'expérience manifeste un sens qui n'est pas le produit de la raison ou de l'entendement. Affirmer le primat de la perception, c'est, en effet, reconnaître une couche de sens préconceptuel comme fondatrice de toutes nos opérations ultérieures de pensée ${ }^{1}$. En décrivant la subjectivité primordiale comme corps, Merleau-Ponty situe la relation au monde dans un commerce avec des significations non transparentes, que le sujet déchiffre sans en être l'auteur car elles s'adressent en lui à un pouvoir impersonnel et anonyme ${ }^{2}$.

Si ses descriptions du corps propre, du sentir, de la motricité, de la parole d'une part (soubassement praxique du théorique), ses analyses de la littérature et de la peinture d'autre part (l'art opérant une réduction qui délivre la dimension originaire du sens), demeurent des voies prépondérantes pour dégager cette sphère du sens incarné à laquelle est adossé tout l'édifice du sens, y compris conceptuel, un chemin plus discret mène à l'examen d'une série de figures marginales du point de vue de la raison : l'enfant, l'halluciné, le primitif ou les figures de l'homme avant la raison, privé de raison, et hors de la raison.

\footnotetext{
${ }^{1}$ Maurice Merleau-Ponty, Phénoménologie de la perception, Paris, Gallimard, 1945, par exemple p. 251 : « Tout le savoir s'installe dans les horizons ouverts par la perception ».

2 Ibid., deuxième partie, chap. 1 : « Le sentir », p. 260 et suivantes.
} 
Ces trois figures, questionnées à de nombreuses reprises dans la Phénoménologie de la perception puis dans le cours de Psychologie et pédagogie de l'enfant prononcé à la Sorbonne de 1949 à 1952, mettent en crise la raison en l'homme, et si nous consentons, avec Merleau-Ponty, à tenir compte de leur expérience respective, nous sommes conduits à réviser l'idée d'une raison universelle, commune à tous les hommes, et légiférant sur la totalité de l'expérience. Reconnaître l'humanité de l'enfant, de l'halluciné et du primitif, et les admettre comme nos possibles, c'est hisser l'articulation entre humanité et raison au rang de problème, moins peut-être, chez Merleau-Ponty, pour se passer de la raison que pour en faire un horizon toujours soumis à la contingence. Au prisme du retournement phénoménologique qui donne le primat au phénomène sur les raisonnements chargés de l'expliquer, ce ne serait donc plus d'un défaut de raison que témoigneraient les figures mentionnées, mais bien d'une mise en défaut de la raison ellemême. Telle est la piste, relativement discrète dans l'œuvre de Merleau-Ponty, que nous souhaiterions explorer.

La réflexion qui va suivre commencera par situer brièvement la critique que Merleau-Ponty adresse à la raison. En quoi consiste-telle? Que vise-t-elle exactement? Puis, nous examinerons l'apport original des analyses consacrées aux trois figures généralement marginalisées par l'approche rationnelle. Enfin, nous nous interrogerons sur le décentrement de la raison opéré en retour par la reconnaissance de ces expériences autres : quelle redéfinition de l'expérience «normale » en découle-t-il ?

\section{1 - Une critique de la transparence rationnelle du monde et de la conscience}

Revenons à la manière dont la critique de la raison s'exprime dans l’«Avant-propos » de la Phénoménologie de la perception:

La plus importante acquisition de la phénoménologie est sans doute d'avoir joint l'extrême subjectivisme et l'extrême objectivisme dans sa notion de monde ou de la rationalité. La rationalité est exactement mesurée aux expériences dans lesquelles elle se révèle. Il y a de la rationalité, c'est-à-dire: les perspectives se recoupent, les perceptions se confirment, un sens apparaît. Mais il ne doit pas être posé à part, transformé en Esprit absolu ou en monde au sens réaliste ${ }^{3}$.

${ }^{3}$ Ibid., « Avant-propos », p. 20. 
Ainsi, la notion de «rationalité » est rabattue sur celle de «monde», interdisant par là une interprétation idéaliste du sens. L'unité des phénomènes, le sens, ne provient pas d'un Esprit absolu séparé des phénomènes pas plus que d'une unification catégoriale par l'entendement. D'autre part, précise Merleau-Ponty, le monde n'est pas à entendre au sens réaliste d'une entité objective séparée. Il n'y a monde qu'aux prises avec un champ phénoménal, c'est-à-dire un horizon d'apparaître. Ainsi, la notion de «monde » est définie par une double négation (« ni »/ «ni ») qui dicte la structure de l'ensemble de l'ouvrage. Le monde est par-delà l'alternative du sens pur et du non-sens : le monde fait sens, c'est-à-dire qu'il y a unification des phénomènes en totalité, et non pas une simple somme d'accidents, mais cette totalité est présupposée par chacune de mes expériences sans être jamais posée pour elle-même et complètement donnée :

Mon point de vue est pour moi bien moins une limitation de mon expérience qu'une manière de me glisser dans le monde entier. Quand je regarde l'horizon, il ne me fait pas penser à cet autre paysage que je verrais si j'y étais, celui-ci à un troisième paysage et ainsi de suite, je ne me représente rien, mais tous les paysages sont déjà là dans l'enchầnement concordant et l'infinité ouverte de leurs perspectives ${ }^{4}$.

En décrivant ainsi l'origine du sens, Merleau-Ponty vise l'idéalisme transcendantal dans la figure de Kant mais surtout de ses successeurs, principalement Lachièze-Rey ${ }^{5}$ et de manière plus ambiguë Cassirer6. Toutefois, la Phénoménologie de la perception ne se situe pas sur le terrain de la distinction kantienne entre raison et entendement, mais, du point de vue de la seule expérience, objecte que la relation au monde ne peut être interprétée comme une opération de catégorisation par l'entendement. La référence à Kant apparaît dans $l^{\prime}$ «Avant-propos » pour distinguer la conception husserlienne de l'intentionnalité du rapport kantien à un objet possible :

Ce qui distingue l'intentionnalité du rapport kantien à un objet possible, c'est aue l'unité du monde, avant d'être posée par la connaissance et dans un acte d'identification expresse, est vécue comme déjà faite ou déjà là7.

\footnotetext{
${ }^{4}$ Ibid., p. 386.

${ }^{5}$ Ibid., par ex. p. 428 et suivantes.

${ }^{6}$ Ibid., par ex. p. 157 et suivantes.

7 Ibid., "Avant-propos », p. 18. La référence kantienne à un « art caché dans les profondeurs de l'âme humaine » est rappelée p. 492.
} 
Loin de l'ignorer, le philosophe de Königsberg aurait également thématisé cette intentionnalité :

Kant lui-même montre dans la Critique du Iugement qu'il y a une unité
de l'imagination et de l'entendement et une unité des suiets avant l'obiet
et aue, dans l'expérience du beau par exemple, ie fais l'épreuve d'un accord
du sensible et du concept, de moi et d'autrui, qui est lui-même sans
concept. Ici le suiet n'est plus le penseur universel d'un sustème d'obiets
rigoureusement liés, la puissance posante aui assuiettit le multiple à la loi
de l'entendement, s'il doit pouvoir former un monde, - il se découvre et se
goûte comme une nature spontanément conforme à la loi de l'enten-
dement

Si la philosophie de Kant peut être réhabilitée, c'est à condition d'étendre à toute la perception, contre les résultats explicites de la Critiaue de la raison pure, les conclusions de la Critique de la faculté de juger :

Mais s'il y a une nature du suiet, alors l'art caché de l'imagination doit conditionner l'activité catégoriale, ce n'est pas seulement le iugement esthétique, mais encore la connaissance qui repose sur lui, c'est lui qui fonde l'unité de la conscience et des consciences ${ }^{9}$.

Certes, Merleau-Pontv n'ignore pas que Kant désigne lui-même l'imagination transcendantale comme la fonction qui permet d'assurer la liaison entre le divers de la sensibilité et les catégories de l'entendement dans La Critiaue de la Raison pure mais, s'inspirant de la ligne critique développée par Heidegger dans le Kantbuch, il considère que Kant ne parvient pas vraiment à remonter à cette unité du sensible et de l'intelligible dont la dualité dérive. En revanche, il reconnaît dans le iugement esthétique kantien «cet accord du sensible et du concept, de moi et d'autrui, qui est lui-même sans concept », au'il voit à l'œuvre dans le percevoir. Il convient, par suite, de généraliser à toute perception et toute connaissance ce que Kant réserve à la seule perception esthétique. Nous ne devons donc pas nous étonner de voir Merleau-Pontv, dans la suite de son œuvre, demander à l'expérience esthétique d'éclairer le sens le plus général de la perception: si toute expérience présuppose, de manière crvptique, l'intervention de l'imagination transcendantale, alors l'expérience esthétique doit seulement nous révéler de manière plus éclatante le sens de toute expérience. S'il faut reconnaître à l'expérience esthétique une fonction épokhale, en ce qu'elle tire le spectateur de la

${ }^{8}$ Ibid., « Avant-propos », p. 20.

${ }_{9}$ Ibid., « Avant-propos », p. 18. 
torpeur associée à l'attitude naturelle, c'est sur le fond d'une perception dont elle ne diffère pas radicalement.

Ainsi, Merleau-Pontv s'appuie, en l'élargissant, sur la notion kantienne $\mathrm{d}^{\prime}$ " imagination transcendantale » en assurant qu'elle replace le suiet de la perception en decà de l'opposition entre diversité sensible et concept. C'est à cet «art caché » de l'imagination que nous devrions l'émergence du sens à même le sensible. Dans le vocabulaire phénoménologique, nous dirions volontiers que tout l'effort de la Phénoménologie de la perception consiste à concevoir les relations entre la phénoménalité et le phénoménal de manière à penser une élaboration du sens qui ne soit pas constitution ${ }^{10}$. Que ie sois « capable par connaturalité de trouver un sens à certains aspects de l'être sans le leur avoir moi-même donné par une opération constituante $»^{11}$ signifie que ie reconnais dans toute perception la présence d'une finalité sans fin, ni assignée, ni assignable. Merleau-Pontv commente en ce sens non-idéaliste l'expression husserlienne de «téléologie de la conscience » dans le passage qui suit la référence à Kant :

Husserl reprend la Critique du Jugement quand il parle d'une téléologie de la conscience. Il ne s'agit pas de doubler la conscience humaine d'une pensée absolue aui, du dehors, lui assignerait ses fins. Il s'agit de reconnaître la conscience elle-même comme proiet $d u$ monde, destinée à un monde qu'elle n'embrasse ni ne possède, mais vers lequel elle ne cesse de se diriger, - et le monde comme cet individu pré-objectif dont l'unité prescrit à la connaissance son but ${ }^{12}$.

Le corrélat de la conscience perceptive - qu'il s'agisse de la chose ou, au-delà, du monde - n'est rien d'obiectif, c'est-à-dire rien de constitué de part en part par l'obiectivation des actes de conscience, rien à quoi la conscience assignerait une fin déterminée, comme lors de la production d'un obiet technique. Reste qu'il s'agit d'un monde, c'est-à-dire d'une unité, d'une entité pourvue de sens, autrement dit $\mathrm{d}^{\prime}$ une finalité que je reconnais sans en être l'auteur ${ }^{13}$.

D'autre part, si sur le plan de la relation au monde l'adversaire est la reconstruction kantienne de l'expérience, du point de vue de la

\footnotetext{
10 Toutefois, l'effort pour contrer l'oubli empiriste de la phénoménalité aboutit dans l'ouvrage à maintenir une distinction abstraite entre la conscience et le perçu, rémanence d'idéalisme que la suite de l'œuvre vise à répudier.

${ }^{11}$ Maurice Merleau-Ponty, Phénoménologie de la perception, op. cit. p. 262.

12 Ibid., « Avant-propos », p. 18.

${ }^{13}$ Notons que, dans La Structure du comportement, c'est la notion de "forme " ou "structure " (Gestalt), par opposition à celle de "signification", qui sert à thématiser cette organisation autochtone que nous reconnaissons cependant comme telle. La Phénoménologie de la perception y fait allusion p. 492.
} 
description de la subjectivité c'est l'approche cartésienne du cogito qui est visée. En témoigne les références critiques à Brunschvicg, Lagneau, Alain, et même à Sartre. Le cogito, en effet, pas plus que la perception du monde, n'offre de présence diaphane. Le cogito tacite décrit dans la Phénoménologie de la perception souligne que la réflexion présuppose structurellement un fond de vie irréfléchie, ce qui exclut jusqu'à l'espoir d'une réflexion purifiée comme celle que thématise Sartre ${ }^{14}$.

Ainsi, la critique adressée à l'intellectualisme est, du double point de vue - objectif et subjectif -, une critique de la transparence rationnelle. En quoi les figures marginales que nous nous proposons d'évoquer jouent-elles un rôle original dans cette critique de la raison?

\section{2 - Des expériences autres que la mienne : comprendre l'altérité}

Commençons par remarquer que les trois figures de l'enfant, de l'halluciné et du primitif sont souvent mentionnées simultanément et aux côtés d'autres expériences auxquelles Merleau-Ponty paraît vouloir les rattacher: l'expérience du malade, de la folie, du rêve, de l'imagination, de l'illusion, et plus généralement l'expérience d'autrui.

Ainsi, dans le chapitre consacré à « la spatialité du corps propre et à la motricité », Merleau-Ponty, se référant à la variété empirique des consciences, énumère «la conscience morbide, la conscience primitive, la conscience enfantine, la conscience d'autrui »15. Dans la dernière section du chapitre sur l'espace consacrée à l'élucidation de notre expérience totale de l'espace, Merleau-Ponty, après avoir décrit l'espace de la nuit, l'espace du rêve, l'espace mythique et l'espace schizophrénique, mentionne le «monde du mythe, [...] du malade et de l'enfant $»^{16}$. Il évoque, en lien avec les premières, l'expérience de l'illusion $^{17}$. Enfin, dans le passage consacré à l'élucidation de l'hallu-

\footnotetext{
${ }^{14}$ Par exemple, Jean-Paul Sartre, La transcendance de l'Ego (1934), Paris, Vrin, 2003, p. 45 : «La réflexion a des limites de droit et de fait. C'est une conscience qui pose une conscience. Tout ce qu'elle affirme sur cette conscience est certain et adéquat. Mais si d'autres objets lui apparaissent à travers cette conscience, ces objets n'ont aucune raison de participer aux caractères de la conscience ». Ou encore Esquisse d'une théorie des émotions (1938), Paris, Le Livre de Poche, 1995, p. 116-117: "La réflexion purifiante de la réduction phénoménologique peut saisir l'émotion en tant qu'elle constitue le monde sous forme de magique ».

${ }^{15}$ Ibid., p. 157.

${ }^{16}$ Ibid., p. 342.

17 Ibid., p. 347.
} 
cination, dans la dernière section du chapitre sur «La chose et le monde naturel », il s'intéresse à l'expérience de l'enfant :

L'enfant met au compte du monde ses rêves comme ses perceptions, il croit que le rêve se passe dans sa chambre, au pied de son lit, et simplement n'est visible que pour ceux qui dorment. Le monde est encore le lieu vague de toutes les expériences. Il accueille pêle-mêle les objets vrais et les fantasmes individuels et instantanés [...]. Avoir des hallucinations et en général imaginer, c'est mettre à profit cette tolérance du monde antéprédicatif et notre voisinage vertigineux avec tout l'être dans l'expérience syncrétique ${ }^{18}$.

Dans ce passage est donc suggéré un lien entre la conscience enfantine, la conscience hallucinée et l'expérience générale de l'imagination. Comment interpréter cette mise en constellation de multiples reprises de notions et d'expériences, semble-t-il, fort différentes les unes des autres?

Tout d'abord, il s'agit d'expériences qui résistent à l'interprétation intellectualiste de la conscience. Montrons-le sur le cas de l'hallucination développé au chapitre sur «La chose et le monde naturel». Cette analyse est l'occasion d'une prise de position méthodologique décisive de la part de Merleau-Ponty. Comme dans le reste de l'ouvrage, le philosophe écarte en effet successivement l'interprétation empiriste et l'interprétation intellectualiste de l'hallucination. Toutes deux accordent à tort un primat à la pensée objective :

En réalité il y a, comme on va voir, ceci de commun que les deux doctrines supposent la priorité de la pensée objective, ne disposent que d'un seul mode d'être, l'être objectif, et cherchent à y introduire de force le phénomène hallucinatoire ${ }^{19}$.

Mais alors que l'empiriste fait de l'hallucination « un contenu sensoriel $»^{20}$, l'intellectualiste dissout $l^{\prime}$ hallucination en faisant de toute conscience une connaissance de ce qu'elle vit. Ainsi, si l'intellectualiste a raison contre l'empiriste lorsqu'il fait une distinction de nature entre l'hallucination et la perception, il a cependant tort de maintenir une conception de la conscience qui transforme tout ce qui est vécu en objet. En effet, pour l'intellectualiste, l'être de la conscience s'identifie à son apparaître ${ }^{21}$; elle ne peut donc rien vivre dont elle n'ait une

\footnotetext{
18 Ibid., p. 401.

19 Ibid., p. 392-393.

${ }^{20}$ Ibid., p. 392.

21 Ibid., p. 393.
} 
intime connaissance. L'objection que Merleau-Ponty lui adresse est la suivante :

[...] si le sujet halluciné connaît objectivement ou pense son hallucination comme telle, comment l'imposture hallucinatoire est-elle possible ?22

Si l'halluciné distingue effectivement, sur le plan du jugement, entre ses perceptions et ses hallucinations, cela ne l'empêche pas, dans le même temps, de croire à ses hallucinations et d'y accorder plus d'importance qu'à ses perceptions : telle est l'imposture hallucinatoire qui reste à comprendre.

La conclusion qu'en tire Merleau-Ponty a une portée méthodologique générale :

Il ne faut plus construire l'hallucination, ni en général construire la conscience d'après une certaine essence ou idée d'elle-même qui oblige à la définir par une adéquation absolue et rend impensables ses arrêts de développement. Quand l'halluciné dit qu'il voit et qu'il entend, il ne faut pas le croire, puisqu'il dit aussi le contraire, mais il faut le comprendre. Nous ne pouvons pas nous en tenir aux opinons de la conscience saine sur la conscience hallucinée et nous considérer comme seuls juges du sens propre de $l^{\prime}$ hallucination ${ }^{23}$.

Ainsi, la fidélité aux phénomènes engage Merleau-Ponty dans une profonde révision de la version intellectualiste de la conscience. Mais ce qui est original ici, c'est que le phénomène qui autorise cette remise en cause est vécu par une altérité a priori inaccessible. Il ne s'agit pas seulement, comme par exemple dans l'introduction de la Phénoménologie de la perception, de dénoncer les préjugés classiques concernant les notions de "sensation», $\mathrm{d}^{\prime}$ « attention » ou de «jugement » pour revenir par une expérience propre à la phénoménalité. Le philosophe, observateur lucide et analyste hypothétiquement impartial, n'hallucine pas; que peut donc signifier, selon le mot de Merleau-Ponty, « comprendre » l'halluciné ? Comment franchir l'obstacle épistémologique d'une expérience dont je ne fais pas l'épreuve en première personne, pour en tirer des conclusions qui ne soient pas arbitraires ? La force de l'argumentaire merleau-pontien consiste précisément à montrer qu'il n'y a pas lieu d'accorder un primat à l'expérience en première personne. Tout vécu est médié - et premièrement, par le langage - et, à ce titre, la conscience ne peut s'assurer de ses résultats qu'en reconnaissant la nécessité d'un passage par l'altérité. Si nous

${ }^{22}$ Ibid., p. 394.

${ }^{23}$ Idem. 
pouvons sans regret abandonner l'idéal chimérique d'une coïncidence de notre propre vécu et de celui d'autrui, c'est précisément parce que la conscience ne coïncide pas elle-même avec son propre vécu. Aussi Sartre, expérimentant sur lui-même, au moyen d'une piqûre de mescaline, le phénomène d'hallucination, n'est-il pas mieux placé que Merleau-Ponty, interrogeant l'expérience d'autrui, pour décrire ce phénomène :

La conscience d'autrui, le passé, la maladie, ne se ramènent jamais dans leur existence à ce que j'en connais. Mais ma propre conscience en tant qu'elle existe et qu'elle s'engage ne se ramène pas davantage à ce que j'en connais. Si le philosophe se donne à lui-même des hallucinations par le moyen d'une piqûre de mescaline, ou bien il cède à la poussée hallucinatoire, et alors il vivra l'hallucination, il ne la connaîtra pas, ou bien il garde quelque chose de son pouvoir réflexif et l'on pourra toujours récuser son témoignage, qui n'est pas celui d'un hallucinant "engagé » dans l'hallucination. Il n'y a donc pas de privilège de la connaissance de soi et autrui ne $m^{\prime} e s t$ pas plus impénétrable que moi-même ${ }^{24}$.

Toutefois, que la connaissance ne se résolve pas dans cette impossible coïncidence ne justifie pas pour autant les interprétations arbitraires. En effet, il n'y aurait d'arbitraire que si autrui était radicalement séparé de moi. Or, plaide Merleau-Ponty, la différence entre la conscience saine et la conscience hallucinée n'est pas celle d'une altérité radicale sans communication possible de l'une à l'autre. Une connaissance de l'autre ainsi que de soi peut émerger dans ce «phénomène à deux pôles $»^{25}$ :

Il ne s'agit ni de le croire sur parole, ni de réduire ses expériences aux miennes, ni de coüncider avec lui, ni de m'en tenir à mon point de vue, mais d'expliciter mon expérience et son expérience telle qu'elle s'indique dans la mienne, sa croyance hallucinatoire et ma croyance réelle, de comprendre l'une par l'autre ${ }^{26}$.

C'est vers une telle connaissance double que tend, aux yeux de Merleau-Ponty, l'anthropologie telle que la pratique Lévi-Strauss :

Il ne s'agit pas pour une anthropologie d'avoir raison du primitif ou de lui donner raison contre nous, il s'agit de s'installer sur un terrain ou nous soyons l'un et l'autre intelligibles, sans réduction ni transposition téméraire [...]. La tâche est donc d'élargir notre raison, pour la rendre capable

\footnotetext{
${ }^{24}$ Ibid., p. 394-395.

${ }^{25}$ Ibid., p. 395.

${ }_{26} \mathrm{Idem}$.
} 
de comprendre ce qui en nous et dans les autres précède et excède la raison ${ }^{27}$.

La compréhension authentique passe ici aussi par une mesure de la plus juste distance entre l'observateur et la société qu'il étudie. Accéder à l'expérience d'autrui, c'est contester qu'il y ait une distance infinie entre nous, mais c'est tout autant refuser de réduire l'expérience d'autrui à une variante - souvent simplifiée - de la mienne.

Les cours de Psychologie et pédagogie de l'enfant manifestent de manière plus approfondie un tout semblable effort. Du point de vue de la méthode, Merleau-Ponty entend ne pas y considérer la conscience enfantine à partir de la conscience adulte. Cette approche négative, qui procède par soustraction à partir de la conscience adulte prise comme point de référence, manquerait l'originalité de la conscience enfantine. Ainsi, comprendre positivement la conduite de l'enfant, c'est accepter qu'elle soit autre. Toutefois, encore une fois, cette altérité ne nous ferme pas la possibilité de comprendre l'enfant. L'autre, pour Merleau-Ponty, n'est jamais le tout autre, l'inconnu radical, qui, à la limite, confinerait à l'ineffable. Ainsi, il ne faut ni considérer l'enfant comme un «adulte en miniature $»^{28}$ ni le considérer comme une nature inaccessible à l'examen, excès entre lesquels a longtemps balancé la psychologie de l'enfant. Dans les deux cas, on donne finalement le monopole de la raison à l'adulte: qu'elle soit considérée comme une variante du "même » ou comme une altérité radicale impénétrable à notre logique et à nos raisonnements, elle est définie négativement à titre de moindre raison ou de déraison.

Merleau-Ponty mobilise a contrario la notion de "structure de la conscience » pour désigner, sans les réifier dans une "nature », des conduites propres à l'enfant, impossibles à l'adulte, mais compréhensibles pour lui. Premièrement, en abandonnant l'idée d'une nature enfantine, la psychologie accepte que son objet d'étude ne soit pas tant l'enfant $\mathrm{qu}^{\prime}$ « un rapport de l'enfant à un être qui n'est plus un enfant $»^{29}$. L'étude de la conscience enfantine doit réintégrer les conduites observées dans un tout social et historique. Elle renseigne donc en définitive tout autant sur la manière dont une société donnée

\footnotetext{
27 Maurice Merleau-Ponty, « De Mauss à Claude Lévi-Strauss » in Signes, Paris, Gallimard, Folio essais, 1960, p. 197-198.

${ }^{28}$ Maurice Merleau-Ponty, Psychologie et pédagogie de l'enfant, cours de Sorbonne 1949-1952, Lagrasse, Verdier, 2001, p. 171.

${ }^{29}$ Ibid., p. 466.
} 
conçoit l'enfance ${ }^{30}$. La notion de «structure » indique le caractère relationnel de la « conscience » étudiée.

Deuxièmement, en se chargeant de dégager une structure, la recherche fuit l'abstraction des approches négatives du comportement infantile. L'étudier de manière seulement soustractive, c'est se condamner à des généralités qui ne prennent pas en compte les histoires individuelles :

On croirait quelquefois, à lire les psychologues, que les conduites enfantines résultent comme par une fatalité de son âge mental. Mais il faut considérer un autre aspect: celui de l'histoire, des événements de l'enfance qui expliquent sa mentalité [...]. Il ne suffit pas de dire que l'enfant n'a pas d'attention, c'est abstrait. Il faudrait dire à quoi il pense, vers quoi son attention est attirée quand il n'a pas $d^{\prime}$ "attention». Il faut ajouter des contenus positifs, mettre en évidence l'aspect fonctionnel de sa conduite, par exemple dire non seulement qu'il symbolise, mais ce qu'il symbolise, comment il le symbolise ${ }^{31}$.

La notion de «structure » n'est ainsi pas à entendre au sens restreint de «forme », mais indique la prise en compte d'études empiriques concrètes à même de décrire positivement les comportements de l'enfant.

Toutefois, les cours donnés à la Sorbonne ne se contentent pas d'approfondir, à propos de l'enfant, les griefs déjà mentionnés à l'égard de la raison. Ils explorent la possibilité d'un parallèle entre différents types de consciences marginales.

\section{2 - Des expériences autres entre elles : comprendre la variété}

Que vaut le parallèle entre l'expérience de l'enfant, d'une part, celles du malade et du primitif, d'autre part ? Merleau-Ponty souligne que la recherche d'une approche adéquate de la conscience enfantine doit finalement dépasser les mêmes obstacles que ceux rencontrés par la psychopathologie et l'ethnologie. Ainsi, Lévy-Bruhl et Blondel auraient eu tendance à décrire, respectivement, la pensée primitive et la conscience morbide comme l'autre radical de la raison, accordant le monopole de la rationalité, l'un à l'adulte civilisé, l'autre au «normal». Toutefois, les conceptions, en la matière, ont changé et de même que «Lévy-Bruhl rejette lui-même la notion de prélogique et

\footnotetext{
${ }^{30}$ Idem.

${ }^{31}$ Ibid., p. 173-174.
} 
parle d'une logique particulière qu'il faut savoir saisir »32, « on en vient à l'affirmation que la conscience normale et la conscience morbide peuvent s'éclairer mutuellement et être connues l'une par l'autre ${ }^{33}$. Si les figures de l'enfant, du primitif et du malade sont comparées à titre d'expériences autres qui nécessitent un abandon de l'hégémonie de la raison pour être comprises, peut-on, pour autant, pousser plus loin le parallèle? En ce point précis, nous rejoignons une deuxième idée maîtresse de la critique de la raison. Selon Merleau-Ponty, la conception intellectualiste de la conscience ruine en fait la diversité des expériences dont elle doit rendre compte. En condamnant à l'apparence les expériences qui ne sont pas celles de l'adulte blanc et sain, une telle approche non seulement les nie, mais a pour conséquence gnoséologique immédiate de toutes les identifier. Ce grief est exposé sans équivoque dans le chapitre "La spatialité du corps propre et la motricité » de la Phénoménologie de la perception; Merleau-Ponty, visant la pensée de Cassirer, y écrit :

Si la conscience est placée hors de l'être, elle ne saurait se laisser entamer par lui, la variété empirique des consciences, - la conscience morbide, la conscience primitive, la conscience enfantine, la conscience d'autrui, - ne peut pas être prise au sérieux, il n'y a rien qui soit là à connaître ou à comprendre, une seule chose est compréhensible, c'est la pure essence de la conscience. Aucune de ces consciences ne saurait manquer d'effectuer le Cogito. Le fou, en arrière de ses délires, de ses obsessions et de ses mensonges, sait qu'il délire, qu'il s'obsède lui-même, qu'il ment, et pour finir il n'est pas fou, il pense l'être ${ }^{34}$.

La conséquence immédiate de ce postulat de transparence de la conscience à elle-même est sans appel - si toutes les maladies mentales ne sont qu'apparences, alors rien ne permet de les distinguer :

L'analyse du sens de la maladie, si elle aboutit à une fonction symbolique, identifie toutes les maladies, ramène à l'unité les aphasies, les apraxies et les agnosies et n'a peut-être aucun moyen de les distinguer de la schizophrénie $e^{35}$.

Dans tous les cas, nous aurions affaire à un même effondrement de l'attitude catégoriale. Or, il est manifeste que si nulle de ces maladies ne saurait se satisfaire d'une explication purement physiologique qui permettrait de localiser avec précision le trouble et d'en rendre

\footnotetext{
32 Ibid., p. 172.

${ }^{33}$ Idem.

${ }^{34}$ Maurice Merleau-Ponty, Phénoménologie de la perception, op. cit. p. 157-158.

35 Ibid., p. 158.
} 
compte causalement, les symptômes de chacune atteignent de manière privilégiée une sphère particulière du comportement :

\begin{abstract}
Même si toute aphasie, observée d'assez près, comporte des troubles gnosiques et praxiques, toute apraxie des troubles du langage et de la perception, toute agnosie des troubles du langage et de l'action, il reste que le centre des troubles est ici dans la zone du langage, là dans la zone de la perception et ailleurs dans la zone de l'action ${ }^{36}$.
\end{abstract}

Ce dédain de la diversité des expériences trouve encore une confirmation dans le Cours de Psychologie et pédagogie de l'enfant au moment de comparer la conscience enfantine aux consciences morbide et primitive. La critique de la raison vise ici moins directement l'incapacité de la conception intellectualiste de la conscience à rendre compte d'expériences différentes, que la référence indépassable à la conscience de l'adulte blanc et sain pour apprécier toute altérité. Héritière d'un préjugé fort commun, la psychologie génétique se donne simultanément comme objet d'étude les consciences infantiles, primitives et morbides ; elle postule ainsi que "l'enfant présente un état archaïque de la conscience adulte, dont le malade présente un état régressif $»^{37}$, et d'autre part, que "l'enfant recommence la croissance de l'humanité ${ }^{38}$. Que valent ces comparaisons ? Selon Merleau-Ponty, pas davantage que nous ne pouvons identifier quelqu'un qui ne sait pas à quelqu'un qui ne sait plus, nous ne pouvons assimiler le comportement d'un individu pleinement adapté à la société dans laquelle il vit à celui d'un individu non adapté. Premièrement, dans le cas de l'aphasie, Merleau-Ponty indique que l'ouverture au sens du nombre existe chez l'enfant qui apprend à compter, quand bien même ses automatismes n'ont pas la sûreté que donne l'héritage d'un apprentissage. Au contraire, l'aphasique conserve des automatismes anciens, est même capable de trouver des automatismes de remplacement pour résoudre une opération, mais il n'est plus capable de compter car il a perdu l'ouverture au nombre :

Ainsi la même action chez le malade paraît en même temps plus aveugle et
plus sûre que chez l'enfant, puisque même dépourvue de sens, elle s'appuie
sur un mécanisme très ancien. Ce mécanisme fait encore défaut chez
l'enfant, l'action est hésitante, mais empreinte de l'insight, la compréhen-
sion intime de l'opération ${ }^{39}$.

36 Idem.
${ }^{37}$ Maurice Merleau-Ponty, Psychologie et pédagogie de l'enfant, op. cit., p. 174.
${ }^{8}$ Ibid., p. 174.
${ }^{39}$ Ibid., p. 175. 
Deuxièmement, s'il peut y avoir un sens à arguer d'une ressemblance entre le primitif et l'enfant ${ }^{40}$, l'hypothèse de récapitulation qui fait de la société primitive l'enfance de la civilisation est contestable, non seulement parce que les statuts de l'enfant et du primitif au sein de leurs sociétés respectives sont rigoureusement différents mais parce que les sociétés dites primitives ont souvent une longue histoire derrière elles. Ainsi, dans les deux cas examinés, le parallélisme forcé repose sur une métaphysique évolutionniste ratiocentrée qui perd de vue l'originalité des expériences à décrire. Ce point manifeste un deuxième aspect de la critique que Merleau-Ponty adresse à la raison, étroitement lié au premier: son impuissance à penser l'altérité premier aspect de la critique - conduit en définitive à un déni de la variété des expériences.

À maintes reprises, les analyses précédentes ont suggéré que la conscience adulte normale trouve, dans ses confrontations aux figures de l'autre, à apprendre sur elle-même. Que nous révèlent, sur notre propre expérience, le phénomène de l'hallucination ou la conscience enfantine?

\section{3 - Des expériences autres ... à la mienne : les leçons de l'altérité}

Ces expériences nous enseignent que nous devons renoncer à une conception intellectualiste de la relation primordiale au monde, y compris dans le cas de l'expérience de l'adulte «normal » et civilisé. L'élargissement de la raison appelé de ses vœux par Merleau-Ponty coïncide avec un rapprochement, au sein de l'expérience en général, des frontières entre réel et imaginaire. Aussi ne doit-on pas être trop surpris de le voir citer l'expérience de l'imagination et de l'illusion aux côtés de celle de la perception, dans les développements consacrés aux différentes consciences que nous avons examinées.

Qu'apprend, par exemple, aux adultes l'expérience de l'enfant? Quelle est la leçon de "cette adhérence aux situations données qui serait le caractère essentiel de la pensée enfantine $»^{41}$. Le monde de l'enfant comporte, certes, plus $\mathrm{d}^{\prime}$ « ultra-choses » ${ }^{42}$ que le nôtre, au

\footnotetext{
${ }^{40}$ Idem. Merleau-Ponty mentionne, chez Lévi-Strauss, l'hypothèse d'un certain «polymorphisme social » commun à l'enfant et au primitif qui expliquerait cette ressemblance.

${ }^{41} \mathrm{Ibid} ., \mathrm{p} .235$.

42 La notion est empruntée à $\mathrm{H}$. Wallon dans son ouvrage Origines de la pensée chez l'enfant.
} 
sens où il comporte plus d'entités dont l'enfant ne peut faire le tour et qui lui apparaissent alors comme des absolus :

L'enfant sait que ses parents ont été enfants d'autres parents, etc., mais il ne le croit que du bout des lèvres, et si l'on pousse l'interrogatoire on s'aperçoit que l'enfant croit qu'il a préexisté à ses parents comme il croit que la «maison" ou le "champ» sont un absolu de la grandeur (l'adulte est grand "comme la maison » etc.). Sur ce plan, l'enfant est incapable de consentir à n'avoir pas été toujours ${ }^{43}$.

Le monde de l'adulte, certes moins riche en «ultra-choses » que celui de l'enfant, continue lui aussi d'en comporter, ne serait-ce que la mort. Si l'homme adulte peut apprendre le système de Copernic et accéder aux notions de temps et d'espace objectifs, ce savoir, qui a ses racines dans la perception, ne saurait naitre hors de tout point de vue. À ce titre, l'expérience de l'homme adulte et celle de l'enfant continuent de communiquer et c'est à cette seule condition qu'une compréhension de l'expérience enfantine est possible.

Que nous signale, en outre, l'hallucination à nous qui n'hallucinons pas? C'est à la faveur de l'examen de l'hallucination que s'opère peut-être le plus explicitement, dans la Phénoménologie de la perception, ce rapprochement du réel et de l'imaginaire ${ }^{44}$ obtenu par un élargissement de l'expérience à ce qui était traditionnellement tenu pour ses marges. Plus que le détail de la conception sartrienne de l'hallucination, ce sont sa méthodologie ainsi que son approche générale de la perception, et conséquemment de l'imagination, qui font l'objet d'une critique de la part de Merleau-Ponty. D'un point de vue méthodologique, nous avons analysé quels étaient ses griefs. Il nous faut désormais préciser les nouvelles relations qu'il instaure entre le réel et l'imaginaire. Revenons à la critique de l'intellectualisme très succinctement présentée précédemment. L'objection que MerleauPonty lui adressait était la suivante :

「...1 si le suiet halluciné connaît obiectivement ou pense son hallucination comme telle, comment l'imposture hallucinatoire est-elle possible ?45

La critique du rationalisme vise explicitement Alain, comme le précisent deux notes ${ }^{46}$, mais atteint également le dispositif sartrien. Du point de vue général du statut de l'image, Merleau-Ponty s'entend

\footnotetext{
${ }^{43}$ Maurice Merleau-Ponty, Psychologie et pédagogie de l'enfant, op. cit., p. 242.

${ }^{44}$ Dans la Phénoménologie de la perception, les chapitres consacrés à «l'espace » et au «cogito» affrontent également cette question, respectivement p. 347-351 et p. 441 et suivantes.

${ }^{45}$ Maurice Merleau-Ponty, Phénoménologie de la perception, op. cit., p. 394.

46 Ibid., note 1 p. 393 et note 1 p. 394.
} 
avec Sartre pour soutenir qu'Alain a tort de dénier toute spécificité à l'image et de ne lui accorder aucun statut positif. Il est nécessaire de rappeler ici la critique sartrienne développée en 1936 dans L'imagination.

Alain, reconduisant l'image à un jugement faux, ne reconnaît aucune consistance à l'image. Il considère que "toute image est perception fausse ${ }^{47}$. Il $\mathrm{n}^{\prime} \mathrm{V}$ a d'autres données sensibles que celles qui sont fournies actuellement par ma perception ; mais selon que ie iuge vrai ou faux, ie constitue ces données en obiets réels ou en fantômes. Ces fantômes sont précisément les images ${ }^{48}$. La perturbation du jugement est, quant à elle, liée aux bouleversements corporels :

Désordre dans le corps, erreur dans l'esprit, l'un nourrissant l'autre, voilà le réel de l'imagination ${ }^{49}$.

Ainsi, il n'v aurait pas de donné imaginaire originaire et irréductible à la perception. Cet abandon de l'image ne convainc pas Sartre car il revient à nier la spécificité de la donnée en images, or, il v a une évidence de l'image pour la conscience. Parmi nos actes de conscience, certains se donnent pour imageants et, pour celui aui se veut fidèle à la description, cette évidence ne saurait être réduite par un quelconque argument ${ }^{50}$. Sartre et Merleau-Pontv conviennent, contre Alain, d'une originalité de la donation imageante qui la rend irréductible à une perception, et s'accordent pour ne pas réduire le phénomène « image » à un iugement. L' « avant-propos » formule en ces termes la critique du rationalisme :

À chaque moment mon champ perceptif est rempli de reflets, de craquements, d'impressions tactiles fugaces que ie suis hors d'état de relier précisément au contexte percu et aue cependant ie place d'emblée dans le monde, sans les confondre iamais avec mes rêveries. Aे chaque instant aussi je rêve autour des choses, j'imagine des obiets ou des personnes dont la présence ici n'est pas incompatible avec le contexte, et pourtant ils ne se mêlent pas au monde, ils sont en avant du monde, sur le théâtre de l'imaginaire. Si la réalité de ma perception n'était fondée que sur la cohérence intrinsèque des "représentations", elle devrait être toujours hésitante, et, livré à mes coniectures probables, ie devrais à chaque moment défaire des sunthèses illusoires et réintégrer au réel des phénomènes aberrants aue j'en aurais d'abord exclus. Il n'en est rien. Le réel est un tissu solide, il

\footnotetext{
${ }^{47}$ Jean-Paul Sartre, L'imagination (1936), Paris, PUF, 2003, p. 133.

${ }^{48}$ Idem.

${ }^{49}$ Ibid., p. 132. Il s'agit d'une citation d'Alain.

50 Ibid., p. 137.
} 
n'attend pas nos iugements pour s'annexer les phénomènes les plus surprenants ni pour rejeter nos imaginations les plus vraisemblables ${ }^{51}$.

Merleau-Pontv admet ici avec Sartre l'évidence anté-prédicative du réel et de l'imaginaire. La critique de la conception iudicative de la perception aboutit à reconnaître que réel et imaginaire se donnent pour ce qu'ils sont, indépendamment de la vraisemblance que mon jugement leur reconnaît, et ne sauraient, à ce titre, être confondus.

Toutefois, le cas exemplaire de l'hallucination manifeste que la communauté de pensée s'arrête là. L'effort de Merleau-Pontv pour rendre compte de l'imposture hallucinatoire fait retomber Sartre dans le camp intellectualiste aux côtés d'Alain. Ainsi, certains éléments de la critique de l'intellectualisme frappent aussi bien la position de Sartre que celle d'Alain, en vertu de la conception de la conscience qu'ils partagent :

Dans l'intellectualisme on cherche à se débarrasser de l'hallucination, à la construire, à déduire ce au'elle peut être à partir d'une certaine idée de la conscience. Le cogito nous enseigne que l'existence de la conscience se confond avec la conscience d'exister, que donc il ne peut rien y avoir en elle sans au'elle le sache, aue réciproquement, tout ce au'elle sait avec certitude, elle le trouve en elle-même, au'en conséauence la vérité ou la fausseté d'une expérience ne doivent pas consister dans son rapport à un réel extérieur, mais être lisibles en elle à titre de dénominations intrinsèques, sans quoi elles ne pourraient jamais être reconnues ${ }^{52}$.

Distinguer absolument, au nom d'une telle idée de la conscience, la conscience percevante et la conscience imageante comme le propose Sartre risque de nous faire manquer le propre de l'hallucination, à savoir le fait que l'on puisse accorder autant, voire plus de réalité, à ce que l'on imagine qu'à ce que l'on percoit. De manière générale, la facon dont Sartre concoit la conscience demeure, via Husserl, héritière du rationalisme cartésien : la conscience entretient une relation privilégiée à elle-même, équivalant à une certitude; au contraire, le transcendant se donne à elle comme probable ${ }^{53}$. Toutefois, lorsqu'il s'agit, dans L'imaginaire, de distinguer la thèse du rêve de celle de la perception, l'accent se déplace. La certitude réflexive que la conscience a d'elle-même trouve un écho dans la manière dont le percu se livre à elle. Sartre ne se contente plus de dire que le percu et l'imaginaire se livrent respectivement à moi de telle sorte que je ne puis les

\footnotetext{
${ }^{51}$ Maurice Merleau-Ponty, Phénoménologie de la perception, op. cit., « Avant-propos », p. 10-11.

52 Ibid., p. 393.

53 Jean-Paul Sartre, La transcendance de l'Ego (1934), Paris, Vrin, 2003, p. 33.
} 
confondre, mais commente ce principe en absolutisant leur distinction : le percu se donne comme une évidence alors que l'imaginaire ne se donne, même dans les cas de fascination extrêmes, que comme crovance. Au savoir implicite aui accompagne toute conscience s'ajoute, dans le cas de la perception, la certitude d'avoir affaire à un "quelque chose » compact, d'inattaquable par le doute. Telle est la définition sartrienne $\mathrm{du}$ "réel ». Cette «évidence» est celle de la présence d'un quelque chose et renvoie en termes husserliens au procédé de « remplissement » de l'intention:

L'évidence propre à la perception n'est donc en aucune facon une impression subiective qui serait assimilable à une spécification de la croyance: l'évidence c'est la présence pour la conscience de l'obiet en personne, c'est le "remplissement» (Erfüllung) de l'intention. Pareillement, pour une conscience réfléchissante dirigée sur une conscience perceptive, la nature perceptive de la conscience réfléchie n'est pas non plus obiet de croyance, c'est un donné immédiat et évident. Il est impossible de sortir de là. Une évidence, c'est une présence. Là où l'évidence est donnée, la croyance n'est ni utile, ni même possible $e^{54}$.

Le rationalisme sartrien consiste à faire du percu une connaissance qui, sans être un jugement, s'établit cependant au-delà du doute. Merleau-Pontv, pour rendre compte du phénomène hallucinatoire, bat en brèche cette distinction entre imaginaire et percu. Si l'hallucination demeure possible, c'est que notre croyance spontanée au monde s'établit, au contraire, en deçà du doute :

Il faut aue, si différente au'elle soit d'une perception, l'hallucination puisse la supplanter et exister pour le malade plus aue ses propres perceptions. Cela n'est possible que si hallucination et perception sont des modalités d'une seule fonction primordiale par laquelle nous disposons autour de nous un milieu d'une structure définie, par laauelle nous nous situons tantôt en plein monde, tantôt en marge du monde [...l. L'enfant met au compte du monde ses rêves comme ses perceptions, il croit que le rêve se passe dans sa chambre, au pied de son lit, et simplement n'est visible aue pour ceux aui dorment. Le monde est encore le lieu vague de toutes les expériences. Il accueille pêle-mêle les obiets vrais et les fantasmes individuels et instantanés, - parce au'il est un individu aui embrasse tout et non pas un ensemble d'obiets liés par des rapports de causalité. Avoir des hallucinations et en général imaginer, c'est mettre à profit cette tolérance $d u$ monde antéprédicatif et notre voisinage vertigineux avec l'être dans l'expérience syncrétique ${ }^{55}$.

\footnotetext{
54 Jean-Paul Sartre, L'imaginaire, op. cit., p. 315.

55 Maurice Merleau-Ponty, Phénoménologie de la perception, op. cit., p. 401.
} 
Conscience perceptive et conscience hallucinée correspondent donc à deux versions possibles de cette même adhésion fondamentale au monde. L'opération perceptive inclut en son sein un principe de faiblesse qui rend possible l'hallucination : elle est ouverte à une corroboration indéfinie, c'est-à-dire iamais définitive. Par rapport à l'héritage husserlien, c'est là faire porter l'accent sur la lecon des esquisses sans surinterpréter, contrairement à Sartre, l'idée selon laquelle, dans la perception, les choses sont présentes en personne à l'intuition :

Dans ce domaine de l'opinion originaire, l'illusion hallucinatoire est possible bien que l'hallucination ne soit iamais une perception et que le monde vrai soit touiours soupçonné par le malade au moment où il s'en détourne, parce que nous sommes encore dans l'être antéprédicatif, et que la connexion de l'apparence et de l'expérience totale n'est qu'implicite et présomptive, même dans le cas de la perception vraie ${ }^{56}$.

Merleau-Ponty ne conteste donc pas l'idée selon laquelle perception et hallucination sont distinctes aux veux de celui aui les vit - c'est le point de départ de son argumentaire - mais il discute la radicalité de l'opposition concernant leur modalité de donation. Sartre, en insistant trop fermement sur la différence entre "évidence» et « crovance » a tendance à sous-estimer la lecon des esquisses et manque ainsi la racine commune à l'imagination et à la perception.

Perd-on alors, dans l'analvse de Merleau-Pontv, la distinction entre conscience perceptive et conscience imageante? De fait, tandis que l'imagination s'établit par un geste de clôture qui enferme dans la subiectivité, la perception, en ouvrant radicalement au monde, s'ouvre à une confirmation possible :

Chez le normal, sans aucune vérification expresse, l'expérience privée se relie à elle-même et aux expériences étrangères, le paysage s'ouvre sur un monde géographique, il tend vers la plénitude absolue. Le normal ne iouit pas de la subiectivité, il la fuit, il est au monde pour de bon, il a sur le temps une prise franche et naïve, tandis aue l'halluciné profite de l'être au monde pour se tailler un monde privé dans le monde commun et bute toujours sur la transcendance du temps ${ }^{57}$.

Ainsi, la frontière entre réel et imaginaire perd sa netteté chez Merleau-Pontv dès la Phénoménologie de la perception parce aue l'on v accède par une même opération qui consiste à s'ouvrir au monde en deçà du jugement. La richesse du perçu n'est plus alors interprétée de

\footnotetext{
56 Idem.

57 Ibid., p. 400.
} 
manière unilatérale, ainsi que Sartre a tendance à le faire en comprenant le réel comme ce qui s'impose à nous, ce qui nous résiste ${ }^{58}$; cette richesse est aussi ce qui fait que le réel n'est iamais tout à fait donné, au'il comporte des latences aui rendent possibles l'hallucination. L'hallucination correspond ainsi à la crispation de la subiectivité sur une série d'apparences qui ne trouvent pas à se relier à la totalité ouverte du monde.

Cette nuance apportée à la perspective sartrienne mérite toute notre attention car les textes ultérieurs de Merleau-Ponty ne cesseront d'approfondir ce point de dissension, mis en évidence sur le cas de $l^{\prime}$ hallucination ${ }^{59}$. Fait notable qui prouve à quel point ces interrogations sont jumelles : le cours de Psychologie et pédagogie de l'enfant, qui explore en profondeur les figures de la raison altérée, est également le texte où est formulée clairement pour la première fois la remise en question de l'opposition sartrienne du réel et de l'imaginaire :

La vraie distinction : réel et imaginaire sont deux consciences ambiguës. La conscience réelle n'est jamais en pleine possession de ce qu'elle pose. Il n'existe pas de critère permettant de reconnaître à coup sûr une image d'une perception. Mais :

- l'irrationnel de la perception est ouvert à une expérience qui le justifie ;

- l'irrationnel de l'imaginaire est fermé, non susceptible de vérification ${ }^{60}$.

\section{Conclusion :}

Défectueuses ou altérées du point de vue d'une raison verrouillée et triomphante, les trois figures interrogées par Merleau-Ponty rendent nécessaires sa redéfinition pour qui prend la peine de s'ouvrir à

\footnotetext{
${ }^{58}$ Par exemple, lorsqu'il oppose le savoir implicite qui accompagne respectivement la conscience perceptive et la conscience imageante : "Une conscience perceptive s'apparaît comme passivité. Au contraire, une conscience imageante se donne à elle-même comme conscience imageante, c'est-à-dire comme une spontanéité qui produit et conserve l'objet en image ». L'imaginaire, op. cit., p. 35.

59 Par ex. Maurice Merleau-Ponty, Le visible et l'invisible, Paris, Gallimard, 1964, notes de travail, mai 1959: "Transcendance de la chose et transcendance du fantasme », p. 242 : "Quand on dit que, - au contraire, - le fantasme n'est pas observable, qu'il est vide, non-être, le contraste n'est donc pas absolu avec le sensible. Les sens sont des appareils à faire des concrétions avec de l'inépuisable, à faire des significations existantes - Mais la chose n'est pas vraiment observable : il y a toujours enjambement dans toute observation, on n'est jamais à la chose même. Ce qu'on appelle le sensible, c'est seulement que l'indéfini des Abschattungen précipite - Or, inversement, il y a une précipitation ou cristallisation de l'imaginaire, des existentiaux, des matrices symboliques ».

${ }_{60}$ Maurice Merleau-Ponty, Psychologie et pédagogie de l'enfant, op. cit., p. 231.
} 
l'altérité qu'elles proposent. Trois points décisifs pour une critique de la raison méritent d'être retenus :

A. Tout d'abord, Merleau-Ponty souligne l'impuissance gnoséologique de la raison. L'explicitation des différentes expériences interrogées résiste à la conception intellectualiste de la conscience. Maintenir une telle perspective conduit finalement à nier des expériences dont on ne parvient pas à rendre compte. Ainsi, pour peu que l'on décide de $s^{\prime} y$ fier, chacune de ces expériences représente une mise en crise de la raison comme pouvoir de connaissance. Elles témoignent en cela de son impuissance gnoséologique.

Ensuite, la prise en considération de l'altérité n'implique pas une compréhension unitaire des différentes formes de conscience. À rebours, s'en tenir à une conception intellectualiste de la conscience ou prendre l'expérience de l'adulte civilisé comme étalon de mesure, c'est perdre la spécificité de chacune de ces expériences. MerleauPonty, en refusant l'interprétation intellectualiste de la conscience, laisse place à une pensée qui prend réellement en compte les différences et l'originalité de chaque expérience.

Enfin, Merleau-Ponty entame dans les textes étudiés une réforme de la conception générale de notre rapport originaire au monde. La découverte que je peux tout de même comprendre ces consciences autres que la mienne aboutit, par contrecoup, à transformer la conception que je me fais du «normal » à partir de la conception intellectualiste de la conscience. La relation de compréhension modifie autant le savoir que nous avons de nous-mêmes que celui que nous prenons de l'autre. Elle oblige à faire des consciences autres nos possibles et entraîne un bouleversement de la conception générale de la conscience - conception qui doit désormais intégrer la possibilité de ces expériences. Le tracé des frontières entre réel et imaginaire se redessine alors sans exclusive. 